\title{
The impact of tobacco smoking on one year patient outcomes at a family medicine residency clinic
}

Scott A. Fields, Ph.D. ${ }^{1}$, Satyakant Chitturi, M.D. ${ }^{1}$, Anoop Kumar, M.D. ${ }^{1}$, Jennifer Rose, D.O. ${ }^{1}$, Andy Tanner, D.O. ${ }^{1}$

Author Affiliations:

1. West Virginia University School of Medicine - Charleston Division, Charleston, West Virginia

The authors have no financial disclosures to declare and no conflicts of interest to report.

\section{Corresponding Author:}

Scott A. Fields, Ph.D.

Professor; Director of Behavioral Science

West Virginia University School of Medicine - Charleston Division

Department of Family Medicine

$5^{\text {th }}$ Floor; Robert C. Byrd Clinical Teaching Center

Charleston, West Virginia 25304

Email: sfields@hsc.wvu.edu 


\section{Abstract}

Objectives: In Appalachia, tobacco smoking rates are significantly higher than the United States average, and many of the comorbidities that exist with tobacco are prominent. The researchers investigated the impact of tobacco smoking on patients for one year of health outcomes at our resident primary care clinic.

Methods: The present study examined outpatient clinic charts and hospital records for a calendar year to determine patient self-reported tobacco rates. Patient medical comorbidities were tracked as well as rates of hospital utilization during the calendar year, and those figures were compared for a group of 90 patients who currently smoke and another group of 90 patients who have never smoked.

Results: Tobacco smokers in the present study were more likely to have a history of several medical comorbidities including COPD, musculoskeletal problems, psychiatric treatment, and substance abuse issues. Additionally, tobacco smokers were more likely to have visited the emergency department and to have been admitted to the hospital during the year.

Conclusions: The problem of tobacco smoking is complex and primary care clinics provide a wealth of information to investigate numerous comorbid concerns. Consistent with prior research, tobacco users in this study were at greater risk for many medical problems.

\section{Keywords}

tobacco, smoking, family medicine, primary care, hospital utilization, health outcomes

\section{Introduction}

Despite all that we know about smoking and the health risks, $17 \%$ of adults still smoke in the United States. In West Virginia, where our resident clinic is located, the smoking rate is $27 \%$, markedly higher than the national average. ${ }^{1}$ That rate makes West Virginia the highest smoking state in the United States and coincides with the second highest death rate for people due to smoking in the country. Further, while the adult smoking rate nationally has gradually declined, West Virginia data indicates that tobacco use has not tapered off significantly over the past 25 years. ${ }^{1}$

In a Morbidity and Mortality Weekly Report by the Centers for Disease Control it was found that smoking is the primary cause for at least $30 \%$ of all cancer deaths, nearly $80 \%$ of deaths due to COPD, and $33 \%$ of cardiovascular disease deaths in the USA. ${ }^{2}$ Thus, the risks of tobacco are numerous, diverse, and lethal. Nicotine in tobacco causes coronary vasoconstriction, increased hypercoagulability, dyslipidemia and endothelial dysfunction. Smoking has been shown to be one of the major causes of numerous types of cancer. In smokers with a smoking related cancer, there are increased chances for getting a second smoking related malignancy. ${ }^{3}$ Nicotine in tobacco also effects insulin sensitivity, increasing the risk for developing type 2 diabetes mellitus. ${ }^{4}$ Smoking has also been found to accelerate bone loss and increase the risk for hip fracture, especially in women. ${ }^{5}$ 
In addition to the human toll, tobacco also takes a great deal of resources in regard to time and money. In the United States, smoking has served as a significant public health burden, particularly in the form of increased use of hospital services. ${ }^{6}$ The Centers for Disease Control and Prevention have estimated that smoking costs the United States $\$ 170$ billion in direct medical expenses and $\$ 156$ billion in lost productivity annually. ${ }^{1}$ Smoking cigarettes has long been associated with the development of chronic medical illness culminating in high morbidity and mortality rates.

A number of studies have displayed that cigarette smoking leads to an increased likelihood of hospitalization. ${ }^{7}$ Data from a selection of US Veterans Affairs outpatient clinics demonstrated that males who reported current or previous smoking on a screening questionnaire were at significantly increased risk of being hospitalized for a primary ambulatory care sensitive condition as compared to never smokers. ${ }^{7}$ Data also indicated that current smokers were at the highest risk of hospitalization when compared to previous smokers and never smokers.

The aim of the present study is to investigate tobacco use variables in an Appalachian primary care resident clinic setting. Prior research has provided a solid baseline for tobacco use in the United States. To assess state-specific current cigarette smoking use among adults, the CDC analyzed data from the 2013 Behavioral Risk Factor Surveillance System (BRFSS). BRFSS is a state-based, telephone survey of non-institutionalized adults conducted annually in all 50 states and several US territories. While the CDC report is very useful as a baseline measure, several limitations exist. First, although most clinic patients today do have a telephone, BRFSS does not include adults without some type of telephone service. ${ }^{1}$ Second, estimates for current smoking prevalence are based on self-report and are not supplemented with medical records which can track smoking status over time at each appointment. Third, the median response rate for the 2013 BRFSS study was below 50\% total which increases the potential for confounds due to non-response bias.

\section{Objectives}

The goal of the present study is to provide information regarding tobacco smoking rates, smoking comorbidities, and hospital utilization rates for smokers and never smokers in our resident primary care center. The present study will involve electronic chart review where patients were specifically asked about current and past smoking during routine primary care medical visits. While it is common knowledge that smoking is harmful to health, this study aims to shed light on how smoking impacts outcomes for current smokers as compared to never smokers over a specified period of time (1 year) in a resident family medicine clinic. As the resident clinic serves a large number of patients in an underserved area, there was particular interest in how outcomes might change in the course of the chart review.

\section{Methods}

\section{Participants}

The records of 180 patient electronic health record (EHR) charts in a primary care resident clinic were reviewed. In addition, the current smoker variable was assessed clinic wide to determine the overall current smoking rate among the 8,024 patients in the dataset.

\section{Inclusion Criteria}


Retrospective chart review occurred for all family practice patients randomly selected who were:

Adults, 18 and older as of March 1, 2012.

Have been a patient in the medical center being studied for at least one year from start date of March 1, 2012 when EHR platform was available.

Current smokers included in the study as indicated by answering current smoker question with "yes."

Never smokers included in the study as indicated by answering never smoked question with "yes."

Have been seen at least twice by primary care provider in the year of the study.

Have had labs ordered at least twice within the year of the study so that outcomes could be compared after one year.

\section{Measures}

A Data Collection Sheet (see Appendix 1) was devised to organize and tabulate relevant variable values. Variables were selected based on relevance to the study's objectives and ease of availability in the clinic's EHR.

\section{Appendix 1: Patient Data Collection Sheet}

1. Patient Number:

2. Age:

3. Gender: Male/Female

4. Race/Ethnicity: Caucasian/African American/Latino/Asian/other

5. Smoker: Current/Former/Never Pack years:

6. Chronic Obstructive Pulmonary Disease (COPD):

7. Asthma:

8. Systolic Coronary Heart Failure (CHF):

9. Diastolic Coronary Heart Failure (CHF):

10. Hypertension (HTN):

11. Coronary Artery Disease (CAD):

12. Hyperlipidemia (HLD):

13. Peripheral Vascular Disease (PVD):

14. Diabetes Mellitus Type 1 (DM-1): 
15. Diabetes Mellitus Type 2 (DM-2):

16. Cerebrovascular Accident (CVA):

17. Cancer

18. Psychiatric: DSM DX: Yes/No Specifics: Anxiety/Depression/ADHD/Bipolar Disorder/Other

19. Substance Abuse: Yes/No Specifics:

20. Hepatitis B:

21. Hepatitis C:

22. Gastro-esophageal Reflux Disorder (GERD) /gastritis:

23. Musculoskeletal pain (MSK):

24. Others:

25. \# ER visits (One Year):

26. \# Hospitalizations (One Year):

27. Total Length of Stay:

28. Total Surgeries (One Year):

29. Total Cholesterol 1 (Chol-1):

30. Total Cholesterol 2 (Chol-2):

31. High Density Lipoprotein 1(HDL)-1:

32. High Density Lipoprotein 2 (HDL)-2:

33. Low Density Lipoprotein 1 (LDL-1):

34. Low Density Lipoprotein 2 (LDL-2):

35. Triglycerides -1 (TG-1):

36. Triglycerides -2 (TG-2):

37. Hemoglobin a1c-1:

38. Hemoglobin a1c-2:

39. Insulin:

40. Oral Diabetes Mellitus Meds:

41. \#Anti-hypertensives:

42. Antidepressants:

43. Statins: 
44. Benzodiazepines (BZD):

45. Opiates:

46. Smoking cessation counseling by provider: Yes/No Specifics:

47. Offered smoking cessation assistance: Yes/ No

48. Body Mass Index (BMI) Initial:

49. Body Mass Index (BMI) Current:

50. Obesity:

51. Blood Pressure (BP) Initial:

52. Blood Pressure (BP) Current:

53. Pulse rate (PR Initial):

54. Pulse Rate (PR Current):

55. Oxygen $(\mathrm{O} 2)$ saturation home:

56. Oxygen $(\mathrm{O} 2)$ saturation Initial:

57. Oxygen $(\mathrm{O} 2)$ later:

58. \#COPD exacerbations:

DATA COLLECTION

1. CAD: Any documentation of stenting, CABG, MI, Catheterization reports revealing CAD not requiring intervention

2. HLD: Will follow ATP III guidelines, minimum interval between CHOL-1 and CHOL-2 to be 6 month interval

3. PVD: Documented imaging / documented arterial ulcer

4. CVA: Medical imaging showing CVA/documentation

5. Substance abuse: Other than smoking, will classify based on prevalence after documenting

6. Obesity: As per documented BMI of 30 or greater

7. Total surgeries: This variable was omitted due to difficulty accurately tracking O/P surgeries in the year

\section{Procedures}

Records were reviewed by all of the investigators retrospectively for a full year for each patient who met the criteria for the study. A power analysis using the G Power 3.1 program revealed that to achieve adequate power (Beta=.05) in the study's MANOVAs, a sample of at least 88 participants would be needed in each group. Therefore, a sample of 90 current smokers and 90 never smokers was obtained from a random list of patients. Each patient had a unique record number that ensured that no person would be included in the study duplicitously. The NextGen and Soarian electronic health record platforms were utilized to gather the data and SPSS was 
used for analyses. Patients were selected from a random numbers list for the analysis. Patients who did not meet the inclusion criteria were excluded from the study. The same procedure was used for both groups in the study until 90 patients were included in each group.

\section{Results}

A frequency analysis of clinic population data indicated an overall smoking rate of $38 \%$ among the 8,024 patients. A random sample of 90 never smokers and 90 current smokers was further analyzed with several univariate and multivariate tests. A p value of .05 was used in all analyses to determine significance.

\section{Table 1: Patient Demographic Descriptive Statistics}

\begin{tabular}{|c|c|c|}
\hline & $\begin{array}{l}\text { Smoking Group } \\
\mathrm{n} \quad(\%)\end{array}$ & $\begin{array}{l}\text { Never Smoker Group } \\
\underline{\mathrm{n}}(\underline{\%)}\end{array}$ \\
\hline Participants & $90(50)$ & $90(50)$ \\
\hline \multicolumn{3}{|l|}{ Gender } \\
\hline Female & $57(63)$ & $54(60)$ \\
\hline Male & $33(37)$ & $36(40)$ \\
\hline \multicolumn{3}{|l|}{ Ethnicity } \\
\hline White & $76(84)$ & $84(93)$ \\
\hline African-American & $13(14)$ & $6 \quad(7)$ \\
\hline Latino & $1 \quad(1)$ & $0 \quad(0)$ \\
\hline \multicolumn{3}{|l|}{ Mental Health History } \\
\hline * Psychiatric Diagnosis & $70(78)$ & $54(60)$ \\
\hline ** Substance Abuse & $16(18)$ & 2 (2) \\
\hline \multirow[t]{2}{*}{ Age (years) } & $(\mathrm{SD})$ & (SD) \\
\hline & $55.2 \quad(8.21)$ & $55.6 \quad(12.75)$ \\
\hline
\end{tabular}

Notes. * Chi square significant $\mathrm{p}<.01 ; * *$ Chi square significant $\mathrm{p}<.001$. 
Demographic data for the two groups is provided in Table 1. Both groups were comprised of mostly female participants and were predominantly white in terms of ethnicity. Age was not significantly different between the groups as both groups had a mean age of about 55 years. Chi square analyses revealed significant differences in the prevalence of psychiatric diagnoses $(\mathrm{p}<$ $.01)$ and substance abuse issues $(\mathrm{p}<.001)$ with the Smoking Group having higher rates of each.

When looking exclusively at the Smoking Group (See Table 2), most patients did not receive formal tobacco treatment at the clinic, $77 \%$ were counseled by their physician regarding smoking and $99 \%$ were counseled by the medical assistant. Finally, the Smoking Group had patients with a long history of tobacco use in terms of mean (M) pack years smoked ( $\mathrm{M}=31$ pack years). Pack years refers to the number of packs of cigarettes a person smokes per day multiplied by the number of years smoked.

\section{Table 2: Smoking Group Treatment Data}

\section{Smoking Group}

n $(\%)$

Counseled by medical assistant about tobacco use

$89(99)$

Counseled by provider about tobacco use

$69(77)$

Received tobacco treatment (NRT, medication)

$7 \quad(8)$

* Pack years smoked

M

(SD)

31.2 (22.49)

Notes: $n=90$. * Pack Year Smoked Range 4-100; Pack year $=$ packs per day smoked times years smoked. 
Table 3 depicts comorbidity and resource utilization records within the year of treatment. Chi square tests for nominal variables revealed significant differences in rates of COPD $(\mathrm{p}<.001)$ and musculoskeletal problems between the two groups $(\mathrm{p}<.01)$. Patients in the Smoker Group demonstrated higher rates of both of those medical concerns. An Independent Samples t-test revealed significantly more medical comorbidities for the Smoking Group vs the Never Smoker groups $(\mathrm{M}=3.80$ vs. 3.23; $\mathrm{p}<.05)$. T-tests for hospital utilization data indicated that the Smoking Group utilized the emergency department significantly more ( $M=1.47$ vs. 0.67$)$, and had a higher rate of hospital admissions $(\mathrm{p}<.05)$ when compared to the Never Smoker Group. In additions, the length of stay was more than a day more for the Smoker Group ( $\mathrm{M}=1.77$ vs. 0.46) which was also significantly different, $\mathrm{p}<.05$.

Table 3: Group Comorbidities and Resource Utilization Data

\begin{tabular}{|c|c|c|c|c|}
\hline & \multicolumn{2}{|c|}{ Smoking Group } & \multicolumn{2}{|c|}{ Never Smoker Group } \\
\hline & $\mathrm{n}$ & $(\%)$ & $\mathrm{n}$ & $(\%)$ \\
\hline * COPD Diagnosis & 36 & $(40)$ & 2 & (2) \\
\hline Asthma & 11 & $(12)$ & 17 & $(19)$ \\
\hline Hypertension & 61 & $(68)$ & 72 & $(80)$ \\
\hline Coronary Artery Disease & 15 & $(17)$ & 16 & $(18)$ \\
\hline Hyperlipidemia & 50 & $(56)$ & 54 & $(60)$ \\
\hline Peripheral Vascular Disease & 5 & (6) & 3 & (3) \\
\hline Diabetes Mellitus Type 2 & 31 & $(34)$ & 33 & $(37)$ \\
\hline Cerebrovascular Accident & 9 & $(10)$ & 4 & (4) \\
\hline Cancer & 10 & $(11)$ & 9 & $(10)$ \\
\hline GERD & 47 & $(52)$ & 37 & $(41)$ \\
\hline ** Musculoskeletal Problems & 67 & $(74)$ & 44 & $(49)$ \\
\hline *** Total Number of Comorbidities & $\mathrm{M}$ & $(\mathrm{SD})$ & $\mathrm{M}$ & (SD) \\
\hline & 3.80 & (1.66) & 3.23 & $(1.50)$ \\
\hline
\end{tabular}

Resource Utilization Data 


$\begin{array}{lcccc}* * * \text { Emergency Dept Visits } & 1.47 & (2.28) & 0.67 & (1.69) \\ \text { *** Hospital Admissions } & 0.70 & (1.37) & 0.28 & (0.83) \\ \text { *** Hospital Days } & 1.77 & (4.62) & 0.46 & (1.75)\end{array}$

Notes: * Chi square significant, $\mathrm{p}<.001 ; * *$ Chi square significant, $\mathrm{p}<.01 ; * * *$ Independent Samples t-test significant, $\mathrm{p}<.05$.

Table 4 depicts one year outcomes for both groups. A series of One Between One Within MANOVAs revealed several significant differences in repeated measures. The Smoking Group had a healthier BMI than the Never Smoker Group $(\mathrm{p}<.001)$. Conversely, the Never Smoker Group had healthier measures on pulse rate $(\mathrm{p}<.01)$ and HDL $(\mathrm{p}<.05)$ than their smoking counterparts. The effect for time was significant in improving both cholesterol and LDL numbers for both smoking and nonsmoking groups $(\mathrm{p}<.01)$. The interaction of time and tobacco group was significant for the cholesterol, HDL, and diastolic blood pressure variables. Cholesterol improved more among the Never Smokers over time $(\mathrm{p}<.01)$ while HDL improved more among the Current Smokers $(\mathrm{p}<.05)$. Diastolic BP also improved over time more for the Current Smokers $(\mathrm{p}<.01)$ reaching the same level of the Never Smokers by the year's end.

\section{Table 4: Smoking and Never Smoker Group One Year Outcome Data}

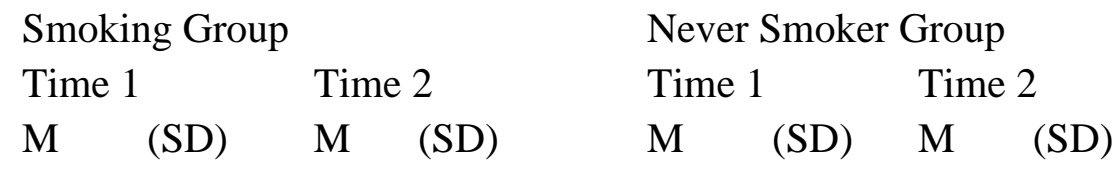

Repeated Measure

$\begin{array}{lccccccccc}{ }^{\mathrm{a} b} \text { Cholesterol } & 186.6 & (47.7) & 185.6 & (55.4) & 193.5 & (41.9) & 172.5 & (37.7) \\ { }^{\mathrm{cd}} \mathrm{HDL} & 45.6 & (12.0) & 46.4 & (13.3) & 51.5 & (15.3) & 49.0 & (15.4) \\ { }^{\mathrm{a}} \mathrm{LDL} & 107.0 & (32.0) & 103.9 & (39.1) & 106.9 & (38.9) & 93.7 & (33.0) \\ \text { Triglycerides } & 169.5 & (119.5) & 173.4 & (128.3) & 171.7 & (79.6) & 151.3 & (81.9) \\ \text { HbA1c } & 7.1 & (1.7) & 7.1 & (2.5) & 6.9 & (1.6) & 6.9 & (1.6) \\ \text { Systolic BP } & 131.1 & (17.7) & 128.7 & (19.0) & 129.2 & (16.1) & 130.0 & (16.1) \\ { }^{\mathrm{b}} \text { Diastolic BP } & 79.7 & (13.9) & 76.6 & (11.5) & 75.6 & (10.3) & 76.6 & (11.7)\end{array}$




$\begin{array}{lcccccccc}\text { e Pulse Rate } & 79.5 & (15.1) & 80.9 & (14.6) & 73.3 & (13.2) & 75.3 & (15.1) \\ { }^{\mathrm{f}} \text { BMI } & 30.8 & (8.0) & 30.3 & (8.3) & 35.1 & (9.3) & 35.0 & (9.3) \\ \text { O2 Saturation } & 97.3 & (2.8) & 96.8 & (2.6) & 97.6 & (2.2) & 97.5 & (2.2)\end{array}$

Notes: One Between One Within MANOVA Results: (a) Effect for Time significant, $\mathrm{p}<.01$; (b) Interaction significant, $\mathrm{p}<.01$; (c) Group difference significant, $\mathrm{p}<.05$; (d) Interaction Significant, $\mathrm{p}<.05$; (e) Group difference significant, $\mathrm{p}<.01$; (f) Group difference significant, $\mathrm{p}$ $<.001$.

\section{Discussion}

The aim of this study was to examine tobacco use, comorbidities and hospital utilization rates among a sample of current smokers and never smokers in our resident Family Medicine clinic located in Appalachia. The $38 \%$ tobacco smoking rate in the clinic was well above the national average of $17 \%$ for tobacco smoking. ${ }^{1}$ Chart records revealed all but one patient in the Smoking group was counseled by either the medical assistant or the doctor about tobacco cessation. This is consistent with the present emphasis on tobacco assessment and tobacco treatment in clinics. Current trends to assess tobacco more frequently stand in contrast to a decade ago when patient tobacco use was not even addressed in many clinic visits. ${ }^{8}$ This would indicate that providers currently buy into the importance of assessing tobacco use in routine medical visits. Moving forward, primary care clinics will continue to play an important role in identifying those with tobacco use disorders and aiding them in their efforts to quit. ${ }^{9}$

Several findings in the present study pointed to the increased risk of other health problems related to smoking. Consistent with prior work on bone density in females, current smokers had higher rates of musculoskeletal issues. ${ }^{5}$ Smoking reduces bone mineral content which puts patients at risk for osteoarthritis and fractures over time. Prior research has also shown that smoking has a negative influence on rheumatoid arthritis, intervertebral disc degeneration and ruptures. ${ }^{10}$ Research has long linked COPD to tobacco smoking. ${ }^{11}$ Well over a third of smokers in the present study had COPD and one per every six patients on average had reported a COPD exacerbation within the year.

Findings on the link between substance abuse, psychiatric diagnoses, and tobacco smoke have been long documented as well, and this was a marked strong finding in the present study. ${ }^{12}$ Prior research has indicated intrinsic (genetic), extrinsic (self-medication) and neuropathway (nicotine receptor) commonalities for those who use tobacco, those who use drugs/alcohol, and those who have longstanding psychiatric issues. ${ }^{13}$ Many primary care clinics nationwide now provide behavioral health services in an integrated care model treating tobacco use in the context of behavioral health issues. This model can be quite helpful, especially in light of evidence that treatments such as nicotine replacement, medication, or focused counseling on tobacco cessation can improve quit rates threefold. ${ }^{8}$

Not all of the outcomes pointed to better results for people who have never smoked. One area where smokers had a healthier outcome was BMI, as smokers were lighter in weight by 4 BMI 
points in comparison to their never smoker counterparts. While BMI is one marker of health, research indicates that a non-smoker of similar height would need to be 100 pounds heavier to have the same health risks as compared to a smoker. ${ }^{14}$ The weight difference among the groups is consistent with prior research linking nicotine to appetite suppression and lowered body weight. ${ }^{15}$

The US Centers for Disease Control has highlighted the negative impact of tobacco smoking on dyslipidemia and cardiovascular functioning. ${ }^{2}$ Repeated measures over the year of study indicated better outcomes in lipid measures (cholesterol, LDL) for both groups. However, never smokers showed more improvement in cholesterol levels throughout the year of the study. Interestingly, while HDL levels were healthier for never smokers, current smokers demonstrated more improvement in HDL levels in the year of study. While this could be explained by another confounding factor, there is also the likelihood that the smoker group had more room for improvement through the course of the year in HDL levels since they had lower levels of HDL from the onset. Regarding cardiovascular variables, patients in the Current Smoker Group were able to improve diastolic blood pressure over time while never smokers did not. In fact, diastolic blood pressure at the end of the study was the same in both groups.

Emergency department (ED) visits and hospital stays can be costly and cumbersome for patients. At first glance, a difference between the two groups of nearly one ED visit and one day in the hospital may not appear to be clinically significant. However, if these figures are extrapolated 10 years into the future, that projects to 10 extra ED visits and 10 extra hospital days per smoker. These higher utilization rates are consistent with prior studies and point to the importance of tobacco cessation early in patient lives prior to health conditions necessitating greater hospital utilization. $^{7,16}$

The present study was not without limitations. First, one concern is the accuracy of self-report in regard to tobacco smoking. This is a potential issue in virtually every study about tobacco use but should be negated somewhat by the present chart review strategy. Second, another limitation of the study was the amount of time patient chart data was collected. The impact of tobacco use is best studied long term, but the clinic in the present study had only made use of the electronic health record (EHR) platform for a little more than one year. Future studies will enable tracking of tobacco use and comorbidities for longer periods of time as the same EHR platform is utilized.

\section{Conclusions}

Future directions for tobacco research should continue to investigate patients in primary care clinics and the impact that smoking has on wellness and subsequent treatment. Smoking rates impacted medical comorbidities and hospital utilization rates in the present study and this all pointed to a continued need for tobacco assessment and cessation services. Strong consideration of behavioral health treatment could also help the high percentage of people who have comorbid addiction and psychiatric issues. The treatment of tobacco use in primary care has the potential to prevent numerous future health concerns, but only if providers are vigilant and patients are willing to work on a shared goal of cessation. 


\section{References}

1. Centers for Disease Control and Prevention. 2014 Behavioral Risk Factor Surveillance System. [cited 2016 May 5]. Available from: http://www.cdc.gov/brfss/.

2. Centers for Disease Control and Prevention. Weekly report on smoking death rates. MMWR Morb Mortal Wkly Rep. 2013;62:15-18.

3. Vineis P, Alvanja M, Buffler P, et al. Tobacco and cancer: recent epidemiological evidence. J Natl Cancer Inst. 2004;21;96(2):99-106.

4. Willi C, Bodenmann P, Ghali WA, et al. Active smoking and the risk of type 2 diabetes: a systematic review and meta-analysis. JAMA. 2007;298:2654.

5. Kanis J, Johnell O, Oden A, et al. Smoking and fracture risk: a meta-analysis. Osteoporos Int. 2004; 16(2):155162.

6. Haapanen-Niemi N, Milunpalo S, Vuori I, et al. The impact of smoking: alcohol consumption, and physical activity on use of hospital services. Am J Public Health. 1999;89(5):691-698.

7. Chew R, Bryson C, Au D, et al. Are smoking and alcohol misuse associated with subsequent hospitalizations for ambulatory care sensitive conditions? J Behav Health Serv Res. 2011;38(1):3-15.

8. Fiore M, Jaen C, Baker T, et al. Treating Tobacco Use and Dependence: 2008 Update, Clinical Practice Guideline. Rockville, MD: U.S. Department of Health and Human Services, 2008.

9. Bornemann P, Eissa A. Strayer S. Smoking cessation: what should you recommend? J Fam Pract. 2016;65(1):22-29B

10. Abate M, Vanni D, Pantalone A, et al. Cigarette smoking and musculoskeletal disorders. Muscles Ligaments Tendons J. 2013;3(2):63-69.

11. Richard P, Gilles H, Alavi Z, et al. Screening for chronic obstructive pulmonary disease in smoking cessation clinic in France. Addiction and Health. 2016;8(1):1-8.

12. Schroeder S, Morris C. Confronting a neglected epidemic: tobacco cessation for persons with mental illnesses and substance abuse problems. Annu Rev Public Health. 2010;31:297-314.

13. Kalman D, Morissette S, George T. Co-Morbidity of smoking in patients with psychiatric and substance use disorders. Am J Addiction. 2005;14(2):106-123.

14. Clark M. Weight concerns in tobacco treatment. In: Mayo Clinic Nicotine Dependence Seminar: Counselor Training and Program Development Manual. Rochester, MN: Mayo Foundation, 2002.

15. Chiolero A, Faeh D, Paccaud D, et al. Consequences of smoking for body weight, body fat distribution, and insulin resistance. Am J Clin Nutr. 2008;87(4):801-809.

16. Ondler C, Hegde G, Carlson J. Resource utilization and health care charges associated with the most frequent ED users. Am J Emerg Med. 2014;32(10):1215-1219. 